\title{
ATP-Permeable Ion Channels Operating in Taste Cells
}

\author{
R. A. Romanov, O. A. Rogachevskaja, A. A. Khokhlov, M. F. Bystrova, and S. S. Kolesnikov \\ Institute of Cell Biophysics, Pushchino, Moscow oblast, 142290 Russia; \\ e-mail: staskolesnikov@yahoo.com
}

DOI: $10.1134 / \mathrm{S} 1990747809030428$

The population of taste bud cells is heterogeneous and includes rounded basal cells, which are progenitors for chemosensory cells, and spindle-like taste cells of three types (type I-type III) that serve the chemosensory function. It has been shown recently that: (i) ATP is the afferent neurotransmitter in the mammalian taste bud, secretion of which finalizes taste transduction [1]; (ii) taste cells of type II release ATP on their stimulation $[2,3]$.

Here we studied ATP release from individual mouse taste cells using a cellular ATP-biosensor. To be triggered by cell depolarization, ATP release did not require an increase in intracellular $\mathrm{Ca}^{2+}$ due to either $\mathrm{Ca}^{2+}$ entry or $\mathrm{Ca}^{2+}$ release, thus arguing against exocytosis as a mechanism predominantly mediating ATP transport across the plasma membrane. Given that cells can alternatively release ATP via ion channels, we aimed to identify and to examine ATP-permeable channels operating in taste cells. The inhibitory analysis of ion currents and ATP secretion suggested that in taste cells, ATP-permeable channels are most likely hemichannels formed by connexins, channel proteins known to mediate gap junction, and/or structurally related pannexins with still debatable cellular functions.

We particularly demonstrated that ATP permeates through ion channels, which transport a voltage-gated (VG) outward current in type II cells, and that there is strong correlation between magnitude of this current and intensity of ATP secretion. These findings suggested that slowly deactivating channels mediating the VG outward current in type II cells also serve as the main conduit of ATP secretion. To verify this pathway, a dependence of ATP release on membrane voltage was studied with different protocols of electrical stimulation of type II cells. The protocols were designed based on predictions of a mathematical model of depolariza- tion-driven electrodiffusion of ATP through a cellular membrane, the process originating transient ATP efflux. Consistently with computer simulations, in experiment, ATP efflux magnitude exhibited bell-like and Langmuir-like dependencies on membrane voltage at relatively short and prolonged electrical stimulations, respectively. Taken together, our theoretical and experimental findings strongly support the idea that slowly deactivating ATP-permeable channels are responsible for the voltage-driven ATP secretion in taste cells of type II. Taking into account that pannexin hemichannels deactivate rapidly, while connexins form slowly deactivating channels, the last are most likely to be the voltage-dependent ATP-permeable channels, which provide the main pathway for stimulus-driven secretion of ATP in taste cells.

The work was supported by the Russian Foundation for Basic Research (project no. 08-04-00033) and by the Program of the President of the Russian Federation (project MK-1332.2008.4).

\section{REFERENCES}

1. Finger, E.T., Danilova, V., Barrows, J., Bartel, D.L., Vigers, A.J., Stone, L., Hellekant, G., and Kinnamon, S.C., ATP Signaling Is Crucial for Communication from Taste Buds to Gustatory Nerves, Science, 2005, vol. 310, pp. 1495-1499.

2. Romanov, R.A., Rogachevskaja, O.A., Bystrova, M.F., Jiang, P., Margolskee, R.F., and Kolesnikov, S.S., Afferent Neurotransmission Mediated by Hemichannels in Mammalian Taste Cells, EMBO J., 2007, vol. 26, pp. 657-667.

3. Huang, Y.J., Maruyama, Y., Dvoryanchikov, G., Pereira, E., Chaudhari, N., and Roper, S.D., The Role of Pannexin 1 Hemichannels in ATP Release and Cell-Cell Communication in Mouse Taste Buds, Proc. Natl. Acad. Sci. USA, 2007, vol. 104, pp. 6436-6441. 